\title{
BENTHIC DIATOMS AS VALUABLE INDICATORS OF ANTHROPOGENIC EUTROPHICATION IN BIOMONITORING OF RIBBON LAKE
}

\author{
OKRZEMKI BENTOSOWE JAKO CENNE WSKAŹNIKI EUTROFIZACJI \\ ANTROPOGENICZNEJ W BIOMONITORINGU JEZIORA RYNNOWEGO
}

\begin{abstract}
In Europe the monitoring of lakes with regard to benthic diatoms is still conducted in line with the European Water Framework Directive. Ribbon lakes are a special case as extremely steep slopes of the lake basin cause their littoral zone to be narrow. The Durowskie ribbon lake was chosen as a model for the assessment of the ecological status of waters based on its Diatom Index. Given its use in recreation, it is under heavy anthropogenic pressure. Physicochemical and biological parameters were monitored in the peak of the vegetational season (July, August) between 2010 and 2018 at 12 varied littoral sites across the full length of the shoreline. This long-term analysis of the Diatom Index, despite showing an improvement in the quality of water, demonstrated the ecological state of Lake Durowskie to be weak (southern, deep part) to moderate (north, shallow part). The taxonomic structure of diatoms (referral and indicator taxa) in phytobenthos communities allowed to show the changes in physicochemical parameters of the environment such as $\mathrm{pH}$, oxygen dissolved in water and its trophic status. Research results are shown in relation to the anthropogenic changes to the lake's direct catchment area and the results of the physicochemical monitoring of waters.
\end{abstract}

Keywords: lake, benthos, Diatom Index, biomonitoring, water quality, physicochemical parameters

\section{Introduction}

Biomonitoring of water bodies in Poland is carried out on the basis of the European Water Framework Directive [1]. According to the Directive, there are four main groups of organisms used for the evaluation of water quality: phytoplankton, phytobenthos together with macrophytes and zoobenthos, and ichthyofauna.

Physicochemical and hydromorphological parameters are to be used in addition to analyses of indicative organisms. Each European country should implement the proper methods on the basis of this general assumptions. Indicators of Environmental Quality should describe the ecological and functional dependence of water ecosystems and consider qualitative and quantitative characteristics of the organisms used. The most common methodology is based on using sensitive species together with those of high tolerance [1-3].

\footnotetext{
${ }^{1}$ Faculty of Biology, Adam Mickiewicz University in Poznan, ul. Umultowska 89, 61-614 Poznań, Poland

*Corresponding authors: messyasz@amu.edu.pl, ewatre@amu.edu.pl
} 
According to the Water Framework Directive lakes are divided into two categories shallow and deep. Post-glacial ribbon lakes, however, are not clearly classified in the Directive which causes debate on which parameters should be used to establish their ecological state. In Wielkopolska region ribbon lakes comprise the majority of all lakes. They are characterized by extremely steep slopes of their lake basin which causes their littoral zones to be narrow. These lakes are used mainly for recreational purposes, which is the main reason for the breakage of the reed zone, specifically due to the creation of beaches, fishing stands and platforms [4].

Measuring the Diatom Index (DI) in accordance with the Water Framework Directive is questionable with regard to ribbon lakes. Their littoral zone is very narrow, thus, often, the only substrate to take samples from is stone. Only benthic diatoms (epilithic communities) are taken into consideration while calculating the DI, with epiphytic communities being ignored. Moreover, diatom communities from the stone were initially described to be more diversified in matter of biodiversity than that of the reed surface [5]. Benthic communities consist of microorganisms living on the sediments of water bodies. Phytobenthos consists of various groups of benthic algae, mainly diatoms. Stones are present in the water during the whole year, which allows the phytobenthic organisms to form stable communities on their surface. The structure of such a community mainly consists of diatoms which are used as indicator species for water quality. The main purpose of research was to determine the changes in water quality in time on the basis of the taxonomical structure of diatoms, expressed by the Diatom Index, as well as to show their compatibility, if such occurs, with chosen physicochemical parameters of water. For the case study Lake Durowskie was chosen, as it has been under yearly monitoring actions since 2010.

\section{Study area}

Lake Durowskie is a post-glacial lake located in the town of Wagrowiec in the Wielkopolska region of Poland. The lake is the last aquatic body in the chain of six lakes of post-glacial origin: Zamkowe, Laskownickie, Grylewskie, Bukowieckie, Kobyleckie and Durowskie itself. These lakes are described as elongated to the north and south, and relatively deep water bodies with steep slopes. The lake is surrounded mainly by buildings and agricultural fields which have been causing eutrophication since 1980. Surface runoff from agriculture pollutes the lake with nitrogen $(\mathrm{N})$, phosphorus $(\mathrm{P})$, and potassium $(\mathrm{K})$ by means of fertilizers, pesticides, and other agricultural chemicals. The high level of pollution in Durowskie Lake comes mainly from other lakes which flow into the Lake Durowskie causing an accumulation of pollutants. Anthropogenic activity, such as the recreational use of the lake and sewage waste from summer houses, also contributes significantly to the state of the lake as it has a negative impact on the quality of water [3, 6].

The shape of the lake and type of usage of shoreline divides the lake into two parts northern and southern. In the northern part the lake is surrounded mainly by forests and agricultural fields with only small amount of fishery stands while on the south part of the lake the town of Wagrowiec is situated along with boat and fishery stands. Also, on this side of the lake there is a beach where recreational activities take place. Due to the type of usage of the shoreline in the northern part of the lake the littoral zone is slightly bigger than in the southern part, however it is still very narrow, according to the profile of ribbon lakes. 
Morphometric characteristics of Lake Durowskie are given in Table 1.

Table 1

Morphometric characteristics of the Lake Durowskie

\begin{tabular}{|c|c|c|}
\hline \multicolumn{2}{|c|}{ Morphometric parameter } & Measurements \\
\hline \multicolumn{2}{|c|}{ Surface area [ha] } & 143.7 \\
\hline \multicolumn{2}{|c|}{ Volume $\left[\mathrm{m}^{3}\right]$} & $11,322,900$ \\
\hline \multicolumn{2}{|c|}{ Maximum depth [m] } & 14.6 \\
\hline \multicolumn{2}{|c|}{ Average depth [m] } & 7.9 \\
\hline \multicolumn{2}{|c|}{ Total catchment area $\left[\mathrm{km}^{2}\right]$} & 236.1 \\
\hline \multicolumn{2}{|c|}{ Direct catchment area [ha] } & 1581.3 \\
\hline \multirow{3}{*}{$\begin{array}{l}\text { Land use of } \\
\text { surrounding } \\
\text { area }\end{array}$} & Agriculture [\%] & 58.26 \\
\hline & Forest [\%] & 33.52 \\
\hline & Urban [\%] & 8.25 \\
\hline
\end{tabular}

a)

$\left.8 \int^{5}\right)_{\substack{\text { Struga } \\ \text { Golani }}}$

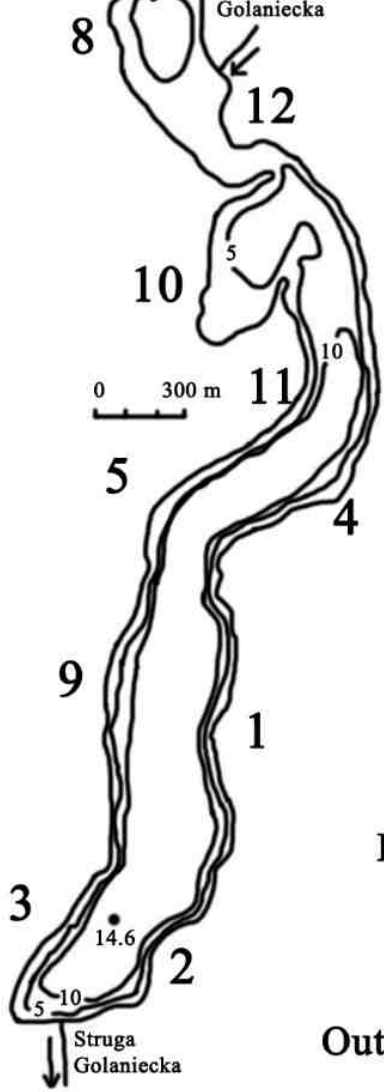

b)
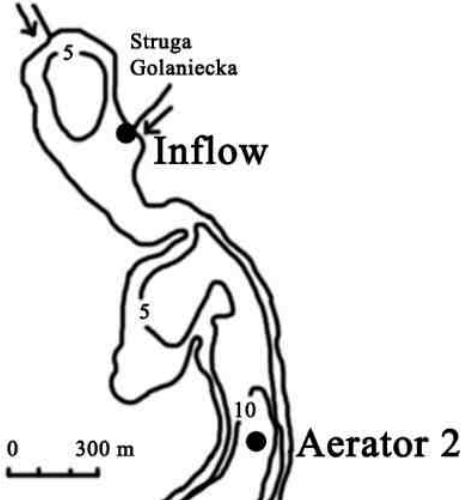

Beach 2 Beach 1

\section{Aerator 1}

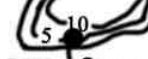

low

Golaniecka

Fig. 1. Map of Lake Durowskie with sampling sites for: a) periphyton (1-12) and b) physicochemical measurements (Aerator 1, Aerator 2, Beach 1, Beach 2, Middle 1, Middle 2, Inflow, Outflow) 


\section{Sampling sites}

Periphyton samples have been taken from 8-12 sites along the shoreline (Fig. 1a) each year since 2010. Between 2010 and 2014 samples were collected from sites 1-8, in 2015 from sites 1-10 and since 2016 from all 12 sites. Sampling sites were chosen along the shoreline in such a manner to represent different types of usage of shores. The northern part of the direct catchment area is covered mainly by forests and agricultural fields while the southern part is situated in the town, site 1 being situated closely to the beach.

Physicochemical parameters were measured on the surface of water in 8 sites showed in Figure 1b.

\section{Methods}

The research was carried out during the summer season (July, August), in years 2010-2018. Physicochemical parameters such as temperature, $\mathrm{pH}$, conductivity and dissolved oxygen (DO) were measured using the multi-parameter water quality meter (YSI 556) at the water surface, with transparency measured using Secchi Disc.

Periphyton species were collected from submerged stones at 8-12 sites along the lake shore. In each case, material was scraped off from a total area of $20 \mathrm{~cm}^{2}$. Samples were preserved using Lugol's iodine solution before taking the samples to the laboratory for analysis. The analysis of the species' composition of diatom communities was carried out according to the Batterbee methods [7]. Identification of species was based on diatomological keys [8-12]. The Diatom Index was used to determine the ecological condition of lake. DI can only be determined when at least 10 species sensitive to trophic level are present in a sample. Trophy Index (TI) and the Index of Referential Species with their standardization are the basic elements to calculate Diatom Index. For an estimation in oxygen saturation, trophy and alkalinity, van Dam's ecological indicators values were used [13].

\section{Results}

\section{Physicochemical quality of water}

The average $\mathrm{pH}$ recorded with the multi-parameter water quality meter along the lake ranged between 7.74 and 8.20 (Fig. 2).

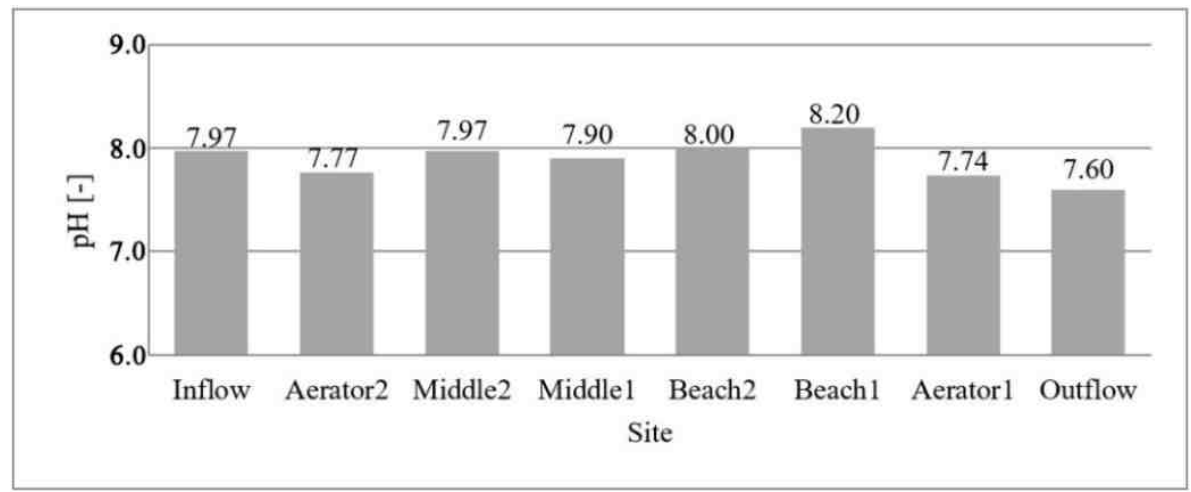

Fig. 2. The average values of $\mathrm{pH}$ parameters measured in the sites in 2018 
The results showed variation in $\mathrm{pH}$ at different parts of the lake, with the middle of the lake described as neutral to alkaline and relatively higher $\mathrm{pH}$ recorded at the two beaches.

From the analysis, the highest dissolved oxygen on the lake was recorded at Beach 1 and Outflow (Fig. 3). Also, Middle 1 and Middle 2 had much higher DO than Aerator 1 and Aerator 2 sites. In all years, the value of dissolved oxygen was higher close to the beaches (Fig. 4). Also, in 2017 the value is higher at Middle 1 and Middle 2.

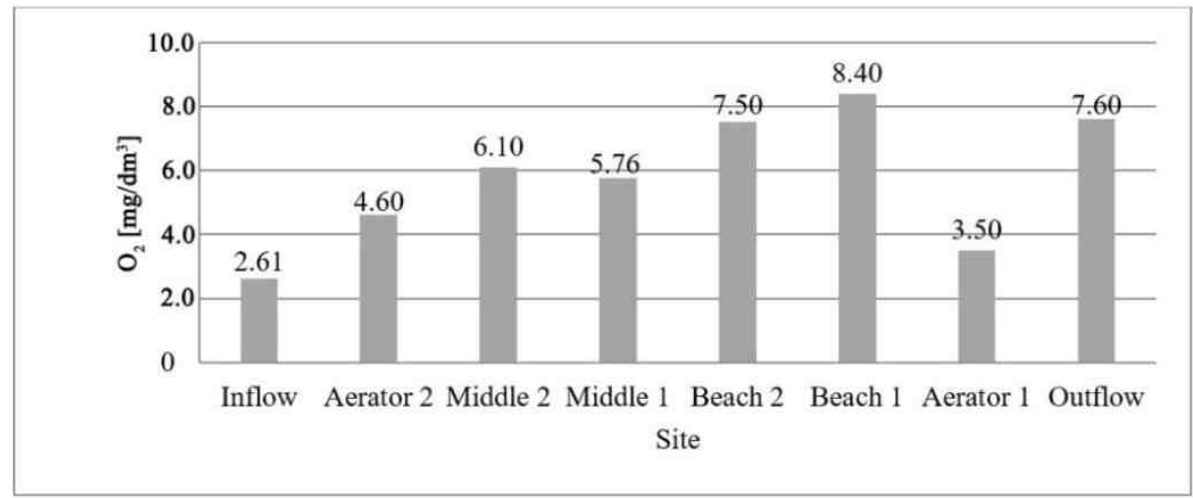

Fig. 3. Dissolved oxygen concentrations on selected sites in Durowskie Lake

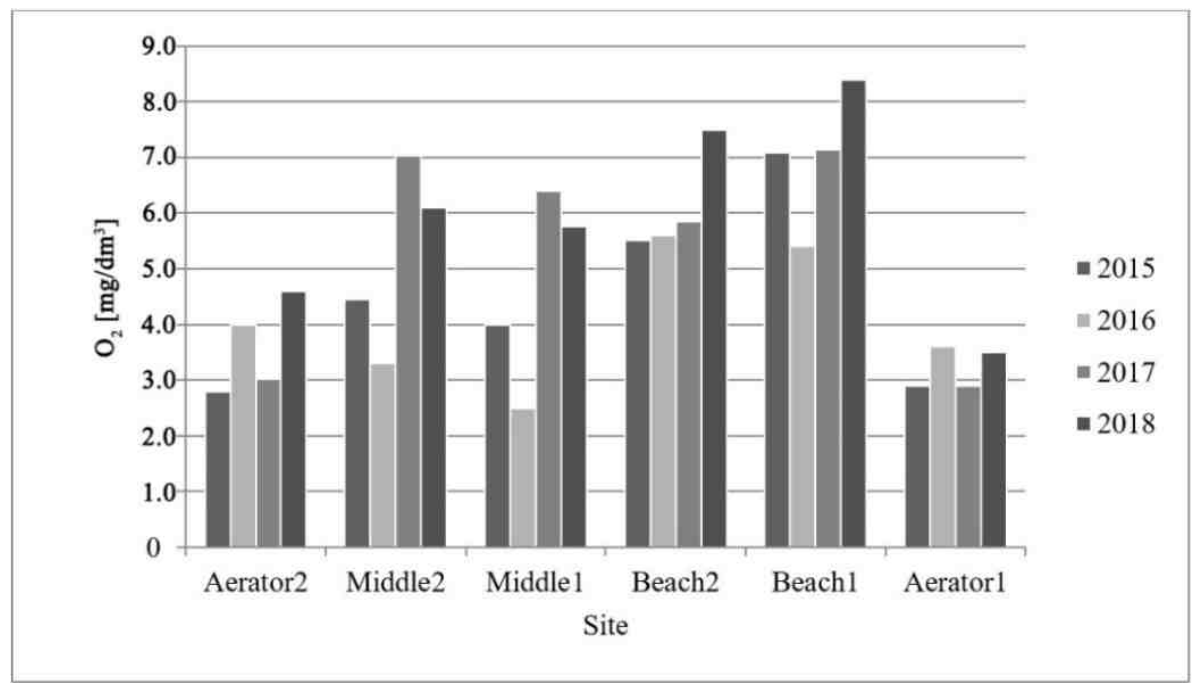

Fig. 4. Trend of dissolved oxygen concentrations on selected sites in Durowskie Lake

\section{Diatom Index}

The analysis of diatom communities attached to stone surface has been done in order to estimate Diatom Index values (Table 2, Fig. 5). The analysis showed occurrence of 104 taxa (Table 3). Species richness was noted between 27 taxa (sites 7 and 12) and 63 taxa (site 11). Only 11 taxa were noted to be present in all studied locations: Achnanthes exigua 
Grun., Achnanthidium minutissimum (Kütz.) Czarnecki, Amphora ovalis (Kutz.) Kutz., Amphora pediculus (Kutz.) Grun., Cocconeis placentula Ehr., Cyclotella radiosa (Grun.) Lemm., Encyonema minutum (Hilse) Mann, Gomphonema olivaceum (Horn.) Breb., Navicula tripunctata (O.F. Müller) Bory, Staurosirella pinnata (Ehr.) Williams \& Round and Ulnaria ulna (Nitzsch) Compere. When analyzing the indicator value of diatom taxa observed in peryphitic communities, it was noted that these were mainly eutrophic species which preferred alkalic waters with high oxygen saturation, of value $75 \%$ and more (Table 3, Fig. 6). 20 species of taxa were found to be dominant, with only 9 of them being dominants in at least three sampling sites. Ecological characteristics of chosen species [11, 13] which were the most numerous in periphytic community of the lake and used as indicators of ecological state of Lake Durowskie, are shown in the Tables 4 and 5. To dominants of high stability of occurrence include: Achnanthes exigua, Achnanthidium minutissimum, A. affinis (Kutz.) Czarnecki, Amphora ovalis, A. pediculus, Cocconeis pediculus Ehr., C. placentula, Cyclotella radiosa, Cymbella affinis Kutz., C. tumida (Brèb.) Van Heurck, Diatoma vulgaris Bory, Encyonopsis microcephala (Grun.) Krammer, E. minutum, Eunotia praerupta Ehr., Fragilaria capucina Desm., F. crotonensis Kitton, Gomphonema olivaceum, G. olivaceoides Hustedt, G. parvulum (Kutz.) Kutz., Staurosirella pinnata. They are mainly species noted in poorly alkaline waters, often consisting calcium and moderate level of concentration of electrolytes. They also occur in anthropogenically changed places but most of them are used as indicators of good water quality.

Table 2

Diatom Index values for each site (2010-2018) with classification of DI values

\begin{tabular}{|c|c|c|c|c|c|c|c|c|c|}
\hline Sites & 2010 & 2011 & 2012 & 2013 & 2014 & 2015 & 2016 & 2017 & 2018 \\
\hline 1 & 0.32 & 0.22 & 0.23 & 0.28 & 0.27 & 0.20 & 0.23 & 0.14 & 0.21 \\
\hline 2 & 0.23 & 0.23 & 0.31 & 0.23 & 0.22 & 0.15 & 0.13 & 0.14 & 0.15 \\
\hline 3 & 0.32 & 0.30 & 0.23 & 0.15 & 0.14 & 0.26 & 0.21 & 0.21 & 0.29 \\
\hline 4 & 0.39 & 0.20 & 0.34 & 0.27 & 0.26 & 0.24 & 0.22 & 0.29 & 0.24 \\
\hline 5 & 0.41 & 0.29 & 0.29 & 0.34 & 0.29 & 0.29 & 0.26 & 0.29 & 0.31 \\
\hline 6 & 0.28 & 0.26 & 0.33 & 0.40 & 0.35 & 0.34 & 0.28 & 0.31 & 0.31 \\
\hline 7 & 0.29 & 0.28 & 0.32 & 0.33 & 0.32 & 0.27 & 0.24 & 0.28 & 0.27 \\
\hline 8 & 0.28 & 0.26 & 0.33 & 0.40 & 0.39 & 0.37 & 0.35 & 0.30 & 0.38 \\
\hline 9 & & & & & & 0.25 & 0.25 & 0.23 & 0.27 \\
\hline 10 & & & & & & 0.32 & 0.27 & 0.28 & 0.32 \\
\hline 11 & & & & & & & 0.31 & 0.30 & 0.31 \\
\hline 12 & & & & & & & 0.27 & 0.27 & 0.31 \\
\hline \multicolumn{5}{|c|}{ Diatom Index value } & \multicolumn{5}{|c|}{ Class } \\
\hline \multicolumn{5}{|c|}{$>0.83$} & \multicolumn{5}{|c|}{ Very good } \\
\hline \multicolumn{5}{|c|}{$0.55-0.82$} & \multicolumn{5}{|c|}{ Good } \\
\hline \multicolumn{5}{|c|}{$0.30-0.54$} & \multicolumn{5}{|c|}{ Moderate } \\
\hline \multicolumn{5}{|c|}{$0.15-0.29$} & \multicolumn{5}{|c|}{ Poor } \\
\hline \multicolumn{5}{|c|}{$<0.15$} & \multicolumn{5}{|c|}{$\mathrm{Bad}$} \\
\hline
\end{tabular}

The Diatom Index shows that the overall water quality is still in the poor to moderate range. Table 2 shows changes in DI for all of the sites for years 2010-2018. For the years 2016-2018 we may see that the Diatom Index increased at almost all sites. However, half of the sampling sites still remain within the "poor quality" boundaries, and most of the sites of moderate state have values close to poor state. 
The average DI for the lake on the basis of results obtained from sites 1-8 in years 2010-2018 (Fig. 5) shows slight changes over the years, with the ecological state changing between poor and moderate water quality.

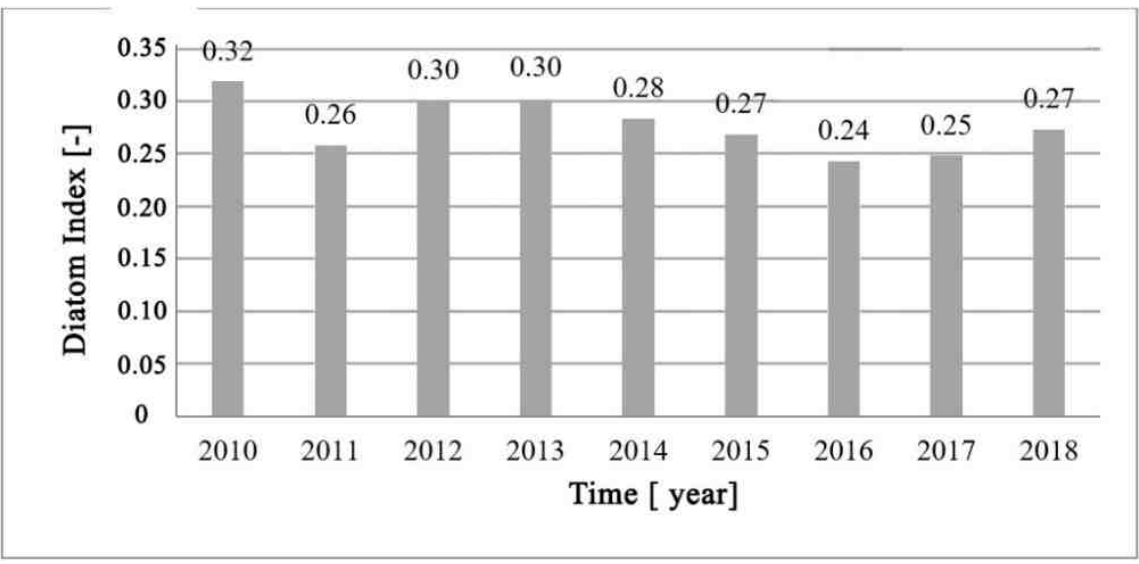

Fig. 5. Mean values for Diatom Index in years 2010-2018

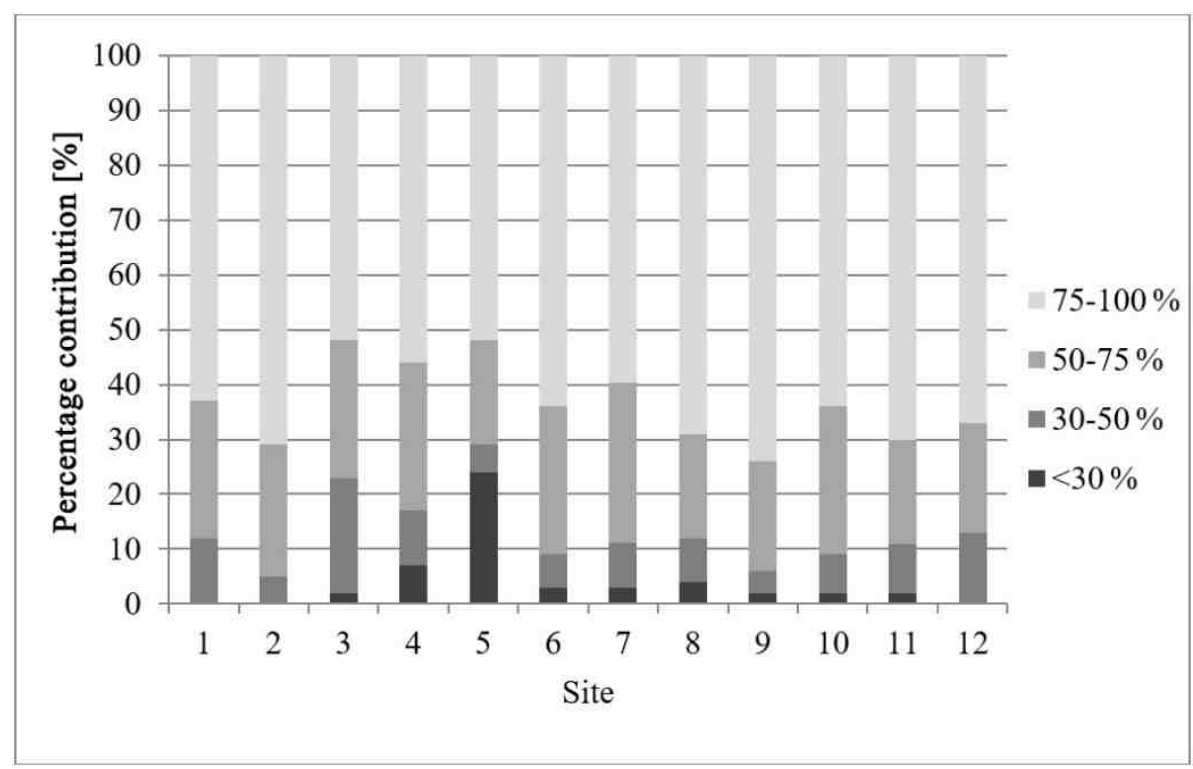

Fig. 6. Periphyton $\mathrm{O}_{2}$ preference for each site

Figure 7 shows the percentage of species with various trophic preferences. The result also indicates the eutrophic state of the Lake Durowskie; however, mesotrophic levels are increasing. 


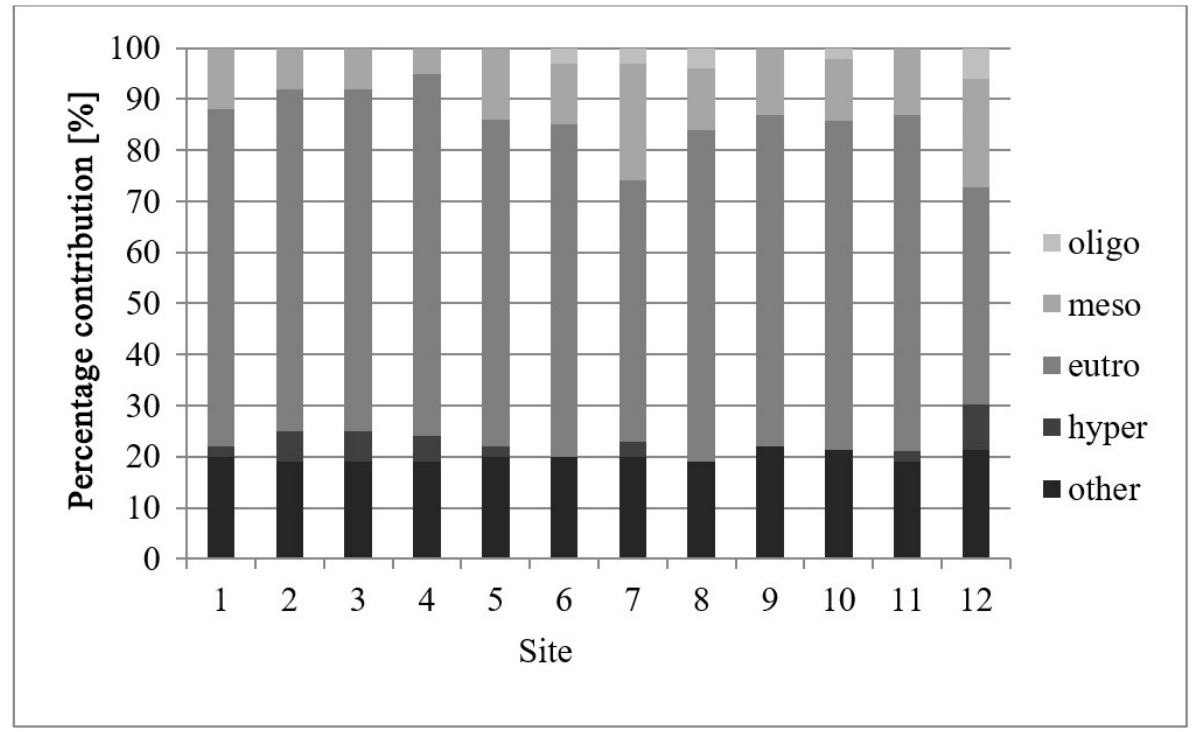

Fig. 7. Periphyton trophic preference for each site

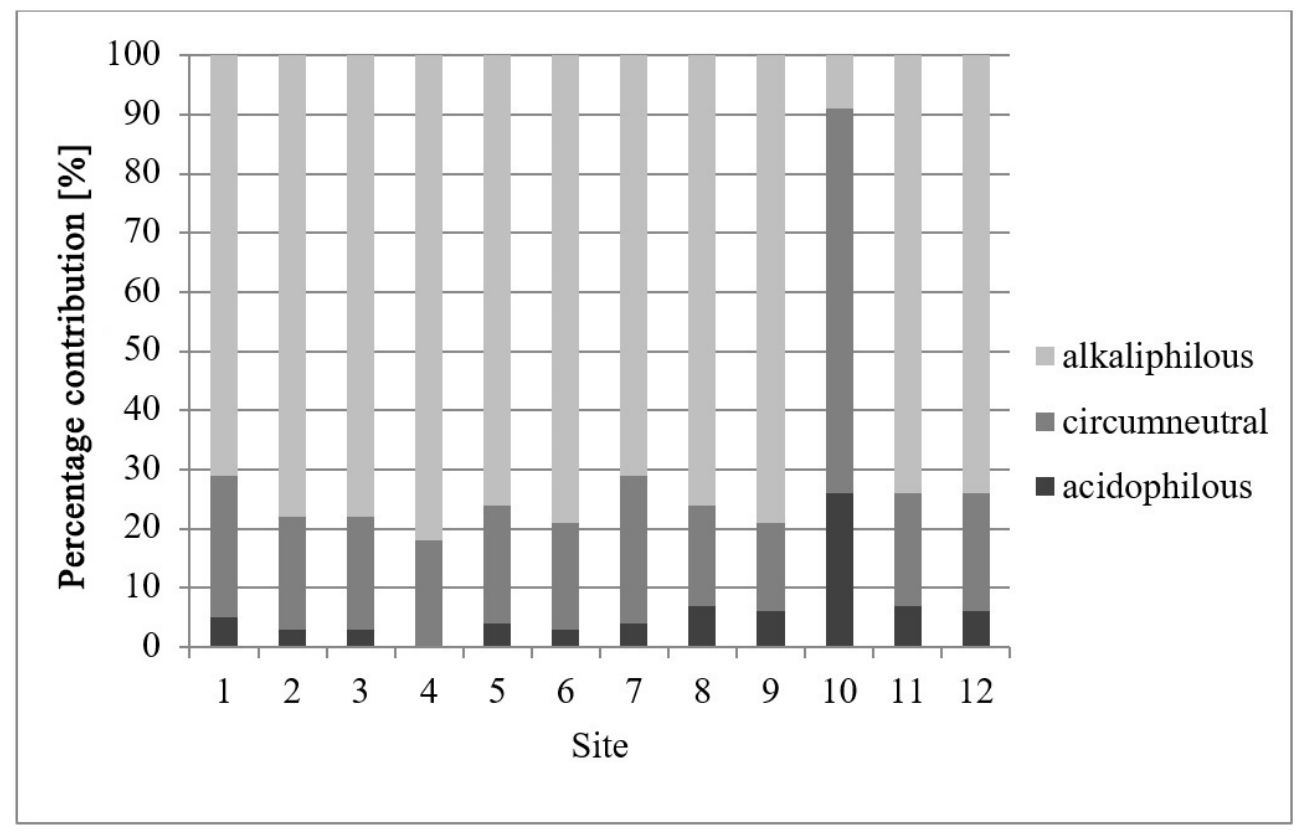

Fig. 8. Periphyton $\mathrm{pH}$ preference for each site

Figure 8 shows different indicator species from acidophilous to alkaliphilous at all 12 sites where samples were taken. All results are shown as percentages. Most sites indicate alkaliphilous species; however, at site 10, the majority of species in this area were noted to 
be circumneutral. Table 3 shows comparison of periphytic diatom species composition with their preferences to $\mathrm{pH}$, oxygen saturation and trophy. Furthermore, Tables 4 and 5 present characteristics of species found to be dominant (Table 4) and occurring rarely (Table 5).

Table 3

Comparison of periphyton species composition in different investigated sites from 2010 to 2018 in Lake Durowskie

\begin{tabular}{|c|c|c|c|c|c|c|c|c|c|c|c|c|c|c|c|}
\hline Diatom taxa / site & 1 & 2 & 3 & 4 & 5 & 6 & 7 & 8 & 9 & 10 & 11 & 12 & $\mathbf{p H}$ & $\mathbf{O}$ & $\mathbf{T}$ \\
\hline Achnanthes exigua Grun. & + & + & + & + & + & + & + & + & + & + & + & + & 4 & 1 & 7 \\
\hline Achnanthidium affinis (Kutz.) Czarnecki & . & . & . & . & . & + & . & + & . & + & . & + & 4 & - & - \\
\hline $\begin{array}{l}\text { Achnanthidium gracillimum (Meister) } \\
\text { Lange-Bertalot }\end{array}$ & . & . & . & . & . & + & . & + & . & . & . & . & 4 & - & 1 \\
\hline $\begin{array}{c}\text { Achnanthidium minutissimum (Kutz.) } \\
\text { Czarnecki }\end{array}$ & + & + & + & + & + & + & + & + & + & + & + & + & 3 & 1 & 7 \\
\hline Amphora ovalis (Kutz.) Kutz. & + & + & + & + & + & + & + & + & + & + & + & + & 4 & 2 & 5 \\
\hline Amphora pediculus (Kutz.) Grun. & + & + & + & + & + & + & + & + & + & + & + & + & 4 & 2 & 5 \\
\hline Asterionella formosa Hassall & + & . & . & . & + & . & . & + & + & + & + & . & 4 & 2 & 5 \\
\hline Aulacoseira granulata (Ehr.) Simon. & . & . & . & . & . & . & . & . & + & . & . & . & 4 & 3 & 5 \\
\hline Caloneis bacillum (Grun.) Cleve & + & + & + & + & . & + & . & . & . & . & + & . & 4 & 2 & 4 \\
\hline Caloneis silicula (Ehr.) Cleve & . & . & . & . & . & . & + & . & . & + & . & + & 4 & 2 & 4 \\
\hline Cocconeis lineata Ehr. & . & . & + & + & . & + & . & . & . & . & . & . & 4 & 3 & 5 \\
\hline Cocconeis pediculus Ehr. & + & + & + & + & + & + & . & . & + & + & + & . & 4 & 2 & 5 \\
\hline Cocconeis placentula Ehr. & + & + & + & + & + & + & + & + & + & + & + & + & 4 & 3 & 5 \\
\hline $\begin{array}{l}\text { Cocconeis placentula var. euglypta (Ehr.) } \\
\text { Grun. }\end{array}$ & . & $\cdot$ & . & . & . & . & . & + & . & . & . & . & - & - & - \\
\hline $\begin{array}{c}\text { Cocconeis pseudolineata (Geitler) } \\
\text { Lange-Bertalot }\end{array}$ & + & . & . & . & . & + & . & . & . & . & + & . & - & - & - \\
\hline Craticula cuspidata (Kutz.) Mann & + & . & . & . & . & . & . & . & . & . & + & . & 4 & 3 & 5 \\
\hline Cyclotella meneghiniana Kutz. & . & + & + & + & + & + & + & + & + & + & + & . & 4 & 5 & 5 \\
\hline Cyclotella radiosa (Grun.) Lemm. & + & + & + & + & + & + & + & + & + & + & + & + & 4 & 2 & 5 \\
\hline Cymatopleura solea (Breb.) Smith & . & . & . & . & + & . & . & . & + & + & . & . & 4 & 3 & 5 \\
\hline Cymbella affinis Kutzing & + & . & + & + & + & + & + & + & + & + & + & + & 4 & 1 & 5 \\
\hline Cymbella cistula (Ehr.) Kirchner & + & + & + & + & + & . & . & . & + & . & + & . & 4 & 2 & 5 \\
\hline Cymbella lanceolata (Ag.) Ag. & . & . & . & . & + & . & . & . & + & + & + & . & 4 & 1 & 7 \\
\hline Cymbella tumida (Breb.) Van Heurck & + & + & . & . & . & . & . & . & + & + & + & . & 4 & 1 & 4 \\
\hline Diatoma tenuis Agardh & . & . & . & . & + & . & . & . & . & + & . & . & 4 & 3 & 5 \\
\hline Diatoma vulgaris Bory & + & . & . & + & + & + & + & + & + & + & + & + & 5 & 2 & 4 \\
\hline Diploneis elliptica (Kutz.) Cleve & . & . & . & . & + & . & . & . & + & + & + & . & 4 & 1 & 3 \\
\hline Encyonema cespitosum Kutz. & . & . & . & . & . & . & + & . & . & . & + & + & - & - & 7 \\
\hline Encyonema elginense (Kram.) Mann & + & . & . & . & . & . & . & . & . & . & + & . & - & - & - \\
\hline $\begin{array}{l}\text { Encyonema leibleinii (Ag.) Silva, Jahn, } \\
\text { Veiga Ludwig \& Men. }\end{array}$ & . & . & . &. & + & . & . & . & + & . & + & . & 4 & 1 & 5 \\
\hline Encyonema minutum (Hilse) Mann & + & + & + & + & + & + & + & + & + & + & + & + & 3 & - & - \\
\hline $\begin{array}{l}\text { Encyonopsis microcephala (Grun.) } \\
\text { Krammer }\end{array}$ & + & + & + & + & + & + & + & + & + & . & + & + & 4 & 1 & 4 \\
\hline Epithemia gibba (Ehr.) Kutz. & + & . & . & . & . & . & . & + & + & + & + & . & 5 & 3 & 5 \\
\hline Epithemia operculata (Ag.) Ruck \& Nakov & . & + & + & . & + & . & . & + & + & + & + & . & - & - & - \\
\hline Eunotia bilunaris (Ehr.) Schaars & . & . & . & . & . & . & + & . & . & . & . & + & 6 & 2 & 7 \\
\hline Eunotia exigua (Brèb. ex Kutz.) Rabenh. & + & . & . & . & + & . & . & . & + & + & + & . & 1 & 2 & 7 \\
\hline Eunotia fabia (Ehr.) Grun. & . & . & . & . & + & . & . & . & + & . & + & . & 2 & 1 & 2 \\
\hline $\begin{array}{l}\text { Eunotia intermedia (Kras. ex Hus.) Nörpel } \\
\text { \& Lange - Bertalot }\end{array}$ & $\cdot$ & . & . & $\cdot$ & • & . & . & . & . & . & . & . & 2 & - & 1 \\
\hline Eunotia lunaris (Ehr.) Grunow & . & . & . & . & . & + & . & . & . & . &. & . & - & - & - \\
\hline Eunotia praerupta Ehr. & + & + & + & . & + & + & + & + & + & + & + & + & 2 & 1 & 2 \\
\hline Eunotia tenella (Grun.) Hustedt & . & . & . & . & . & . & . & . & . & . & . & . & 2 & 1 & 1 \\
\hline
\end{tabular}




\begin{tabular}{|c|c|c|c|c|c|c|c|c|c|c|c|c|c|c|c|}
\hline Diatom taxa / site & 1 & 2 & 3 & 4 & 5 & 6 & 7 & 8 & 9 & 10 & 11 & 12 & pH & $\mathbf{O}$ & $\mathbf{T}$ \\
\hline $\begin{array}{c}\text { Fragilaria amphicephaloides } \\
\text { Lange-Bertalot }\end{array}$ & & & . & & & . & & & . & + & . & . & - & - & - \\
\hline Fragilaria capucina Desm. & + & + & + & + & + & + & . & . & + & + & + & . & 3 & - & 3 \\
\hline Fragilaria constricta Ehr. & $\cdot$ & $\cdot$ & . & $\cdot$ & . & . & . & . & . & + & . & . & 2 & - & 1 \\
\hline Fragilaria crotonensis Kitton & + & + & + & + & + & + & + & + & + & . & + & + & 4 & 2 & 3 \\
\hline Fragilaria exigua Grun. & & & $\cdot$ & & $\cdot$ & . & & + & . & . & . & . & - & - & - \\
\hline Fragilaria vaucheriae (Kutz.) Petersen &. & . & . & . & . & . & . & . & . & . & + & . & - & - & - \\
\hline Gomphonema acuminatum Ehr. & + & 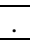 & . & + & + & + & . & + & + & + & + & + & 4 & 2 & 5 \\
\hline $\begin{array}{l}\text { Gomphonema angustatum (Kutz.) } \\
\text { Rabenhorst }\end{array}$ & $\cdot$ & . & . & . & $\cdot$ & $\cdot$ & . & . & . & + & . & . & - & - & - \\
\hline Gomphonema angustum Ag. & . & . & . & . & . & . & . & . & . & + & . & . & 4 & 1 & 1 \\
\hline Gomphonema augur Ehr. & + & . & . & . & . & . & . & . & . & . & + & . & 4 & 1 & 4 \\
\hline Gomphonema gracile Ehr. & + & . & . & . & + & . & . & . & + & . & + & . & 3 & 1 & 3 \\
\hline Gomphonema intricatum Kutz. & . & . & . & . & + & . & . & . & + & . & + & . & - & - & - \\
\hline Gomphonema micropus Kutz. & . & . & . & . & + & . & . & . & + & + & + & . & 4 & 2 & 5 \\
\hline Gomphonema olivaceum (Horn.) Breb. & + & + & + & + & + & + & + & + & + & + & + & + & 5 & 2 & 5 \\
\hline Gomphonema parvulum (Kutz.) Kutz. & + & - & . & . & + & . & . & + & . & + & + & . & 3 & 4 & 5 \\
\hline Gomphonema truncatum Ehr. & . & $\cdot$ & $\cdot$ & $\dot{\square}$ & . & . & . & . & . & . & . & . & 4 & 2 & 4 \\
\hline Hantzschia amphioxys (Ehr.) Grun. & . & & 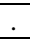 & -1 & + & - & 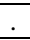 & . & + &. & + & . & 3 & 2 & 7 \\
\hline $\begin{array}{c}\text { Hippodonta capitata (Ehr.) Lange-Bertalot, } \\
\text { Metz. \& Witk. }\end{array}$ & . & + & + & . & + & + & + & + & + & + & + & + & 4 & 3 & 4 \\
\hline $\begin{array}{c}\text { Lemnicola hungarica (Grun.) Round \& } \\
\text { Bassow }\end{array}$ & $\cdot$ & . & . & + & . & . & . & + & . & . & . & . & 4 & 4 & 6 \\
\hline Mastogloia smithii Thwaites ex Smith & . & & . & $0^{\circ}$ & . & + & & $0_{0}$ & . & . &. & . & 4 & - & - \\
\hline Mayamaea agrestis (Hust.) Lange-Bertalot & . & . & . & . & + & . & . & + & . & . & . & . & 3 & - & - \\
\hline Meridion circulare (Grev.) Ag. & . & + & + & + & . & + & . & . & . & + & . & . & 4 & 2 & 7 \\
\hline Navicula cincta (Ehr.) Ralfs & + & + & . & + & + & . & . & + & + & + & + & . & 4 & 3 & 5 \\
\hline Navicula cryptocephala Kutz. & + & . & . & . & + & . & . & . & + & + & + & . & 3 & 3 & 7 \\
\hline Navicula dicephala Ehr. & + & . & . & . & . & 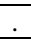 & 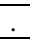 & . & . & . & + & . & - & - & - \\
\hline Navicula gregaria Donkin & . & . & . & . & . & + & + & + & . & . & . & . & 4 & 4 & 5 \\
\hline Navicula oblonga (Kutz.) Kutz. &. & . & . & . & . & . & . & . & . & + & . &. & - & - & - \\
\hline Navicula radiosa Kutz. & + & + & + & + & + & + & . & + & + & + & + & + & 3 & 2 & 4 \\
\hline Navicula reinhardtii (Grun.) Grun. & 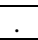 & + & + & + & . & . & . & . & . & + & . & . & 5 & 2 & 5 \\
\hline Navicula tripunctata (O.F. Muller) Bory & + & + & + & + & + & + & + & + & + & + & + & + & 4 & 2 & 5 \\
\hline Navicula veneta Kutz. &. & . & . & + & . & . & + & . & . & . & . & . & - & - & - \\
\hline Navicula viridula (Kutz.) Ehr. & 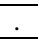 & 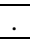 & - & 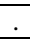 & + & . & . & . & + & . & + & . & 4 & 2 & 5 \\
\hline Nitzschia acicularis (Kutz.) Smith & + & + & + & + & . & . & . & . & . & . & . & . & 4 & 4 & 5 \\
\hline Nitzschia amphibia Grun. & + & $\cdot$ & . & + & + & . & . & . & + & + & + & . & 4 & 3 & 5 \\
\hline Nitzschia incospicua Grun. & - & . & . & . & . & + & . & . & . & + & + & . & 4 & 3 & 5 \\
\hline Nitzschia micropus Kutz. & + & . & . & . & . & . & . & . & . & . & + & . & - & - & - \\
\hline Nitzschia palea (Kutz.) Smith & + & + & + & + & + & . & . & + & . & . & + & . & 3 & 4 & 6 \\
\hline Nitzschia paleacea (Grun.) Grun. & + & + & + & + & . & + & . & . & . & . & + & . & 4 & 3 & 5 \\
\hline Nitzschia recta Hantz. ex Rabenh. & + & 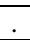 & 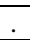 & 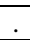 & + & + & . & + & + & . & + & + & 4 & 2 & 7 \\
\hline Nitzschia sigmoidea (Nitzsch) Smith & & + & + & + & + & 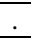 & . & . & + & + & + & . & 4 & 3 & 5 \\
\hline $\begin{array}{l}\text { Pantocsekiella ocellata (Pant.) } \\
\text { Kiss \& Acs }\end{array}$ & + & + & + & + & + & + & . & + & + & . & + & + & 4 & 1 & 4 \\
\hline Pinnularia gibba (Ehr.) Muller & . & . & . & . & . & . & . & . & . & . & . & + & 3 & 3 & 7 \\
\hline Pinnularia maior (Kutz.) Rabenh. & . & . & . & . & . & . & . & + & . & . & . & . & - & - & - \\
\hline Pinnularia viridis (Nitzsch) Ehr. & 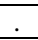 & + & + & + & . & + & . & . & . & . &. & . & 3 & 3 & 7 \\
\hline Placoneis elginensis (Greg.) Cox &. & . & $\cdot$ & $\cdot$ & . & + & . & + & . & . & . & . & - & - & - \\
\hline Placoneis gastrum (Ehr.) Kutz. & . & ${ }^{\circ}$ & . & . & . & . & . & . & . & + & . & . & 4 & 4 & 5 \\
\hline Placoneis placentula (Ehr.) Meres. &. & . & . & . & + & . & . & . & + & + & + & . & 4 & 2 & 5 \\
\hline Planothidium ellipticum (Cleve) Edlund & & & & & . & + & & 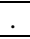 & . & . & - & . & 4 & - & - \\
\hline $\begin{array}{c}\text { Planothidium lanceolatum (Breb. ex Kutz.) } \\
\text { Lange-Bertalot }\end{array}$ & + & + & + & + & . & + & + & + & . & . & + & + & 4 & 3 & 5 \\
\hline
\end{tabular}


Benthic diatoms as valuable indicators of anthropogenic eutrophication in biomonitoring of ribbon lake 719

\begin{tabular}{|c|c|c|c|c|c|c|c|c|c|c|c|c|c|c|c|}
\hline Diatom taxa / site & 1 & 2 & 3 & 4 & 5 & 6 & 7 & 8 & 9 & $\mathbf{1 0}$ & 11 & 12 & pH & $\mathbf{O}$ & $\mathbf{T}$ \\
\hline $\begin{array}{l}\text { Planothidium rostratum (Østrup) } \\
\text { Lange-Bertalot }\end{array}$ & . & & . & + & . & . & & & . & . & . & . & - & - & - \\
\hline Platessa conspicua (Mayer) Lange-Bertalot & & & & & . & . & & + & . & . & $\cdot$ & . & - & - & - \\
\hline $\begin{array}{l}\text { Rhoicosphenia abbreviata (Ag.) } \\
\text { Lange-Bertalot }\end{array}$ & . & . & + & . & . & . & . & . & . & . &. & + & 4 & 2 & 5 \\
\hline Stauroneis phoenicenteron (Nitzsch) Ehr. & + & + & + & + & . & + & & + & . & . & + & . & 3 & 3 & 4 \\
\hline Staurosira construens Ehr. & . & + & + & + & + & . & . & . & + & . & . & . & 4 & 1 & 4 \\
\hline $\begin{array}{l}\text { Staurosirella martyi (Herib.-Joseph) } \\
\text { Morales \& Manoylov }\end{array}$ & . & . & . & & + & . & & & + & + & + & . & - & - & - \\
\hline $\begin{array}{c}\text { Staurosirella pinnata (Ehr.) Williams \& } \\
\text { Round }\end{array}$ & + & + & + & + & + & + & + & + & + & + & + & + & 4 & 1 & 7 \\
\hline Stephanodiscus astraea (Kutz.) Grun. & . &. & $\cdot$ & $\cdot$ & + & . & . & + & + & + & + & . & - & - & - \\
\hline Stephanodiscus hantzschii Grun. & . & + & + & 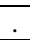 & . & . & . & + & . & . & . & . & 5 & 4 & 6 \\
\hline Surirella minuta Breb. ex Kutz. & $\dot{x}$ & + & + & + & 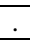 & . & $i$ & . & . & . & . & . & 4 & 3 & 5 \\
\hline Surirella ovalis Breb. & . & 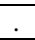 & . & 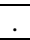 & + & . & . & . & + & . & + & . & 4 & 4 & 5 \\
\hline Ulnaria capitata (Ehr.) Compere & . & + & + & + & . & - & - & + & . & . & 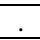 & . & - & - & - \\
\hline $\begin{array}{l}\text { Ulnaria delicatissima var. angustissima } \\
\text { (Grun.) Aboal \& Silva }\end{array}$ & + & + & + & + & + & + & . & + & + & + & + & . & 4 & 2 & 7 \\
\hline Ulnaria ulna (Nitzsch) Compere & + & + & + & + & + & + & + & + & + & + & + & + & 4 & 3 & 7 \\
\hline Tabellaria fenestrata (Lyng.) Kutz. & + & . & . & 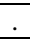 & . & + & + & + & . & + &. & . & 3 & 1 & 2 \\
\hline
\end{tabular}

1-12 - sites; + - present, - not found; $\mathrm{pH}: 1$ - acidobiontic (optimal occurrence at $\mathrm{pH}<5.5$ ); 2 - acidophilous (mainly occurrence at $\mathrm{pH}<7$ ); 3 - circumboreal (mainly occurring at $\mathrm{pH}$ - values about 7); 4 - alkaliphilous (mainly occurring at $\mathrm{pH}>7$ ); 5 - alkalibiontic (exclusively occurring at $\mathrm{pH}>7$ ); 6 - indifferent (no apparent optimum). O: 1 - continuously high (about $100 \%$ saturation); 2 - fairly high (above $75 \%$ saturation); 3 - moderate (above $50 \%$ saturation); 4 - low (above $30 \%$ saturation); 5 - very low (about $10 \%$ saturation). T: 1 - oligotraphenic; 2 - oligo-mesotraphenic; 3 - mesotraphenic; 4 - meso-eutraphenic; 5 - eutraphenic; 6 - hypereutraphenic; 7 - oligo-hypereutraphenic

Ecological characteristics of dominant periphyton species (based on [14-18])

Table 4

\begin{tabular}{|c|c|}
\hline Dominant species & Comment \\
\hline Achnanthes exigua Grun. & $\begin{array}{c}\text { Lives in calcareous lakes, can survive with low light intensity, is able to live until } \\
\text { critical threshold ( } \beta-, \alpha \text {-mesosaprob). }\end{array}$ \\
\hline $\begin{array}{c}\text { Achnanthidium } \\
\text { minutissimum (Kutz.) } \\
\text { Czarnecki }\end{array}$ & $\begin{array}{l}\text { Can live both in oligotrophic an eutrophic conditions, is not able to live in very acidic } \\
\text { conditions or environments with low electrolyte habitats, already under } \alpha-, \beta \text { - } \\
\text { mesosaprobie vitality strongly decreases. }\end{array}$ \\
\hline $\begin{array}{l}\text { Achnanthidium affinis } \\
\text { (Kutz.) Czarnecki }\end{array}$ & $\begin{array}{c}\text { Can live in oliogosaprobic, oligotrophic to eutrophic conditions weak alkaline } \\
\text { conditions, mostly found in calcareous conditions. Often occurs in habitats with high } \\
\text { electrolyte content. }\end{array}$ \\
\hline $\begin{array}{l}\text { Amphora ovalis (Kutz.) } \\
\text { Kutz. }\end{array}$ & $\begin{array}{l}\text { Appear frequently but not very numerous in oligo- to eutrophic water bodies. } \\
\text { Medium to high electrolyte content, higher trophical states are preferred. }\end{array}$ \\
\hline $\begin{array}{l}\text { Amphora pediculus } \\
\text { (Kutz.) Grunow }\end{array}$ & $\begin{array}{l}\text { Lives in oligosaprobic and } \beta \text {-mesosaprobic habitats, very broad trophic spectrum, } \\
\text { missing in conditions with low electrolyte contents and acidic conditions. }\end{array}$ \\
\hline Cocconeis pediculus Ehr. & $\begin{array}{l}\text { Lives in medium to high trophic state, does not appear in conditions with low } \\
\text { electrolyte content, circumneutral to acidid conditions or oligotrophic habitats. }\end{array}$ \\
\hline Cocconeis placentula Ehr. & $\begin{array}{c}\text { Broader spectrum than Cocconeis pediculus, in contrast appears in conditions of low } \\
\text { electrolyte content and medium trophic levels. }\end{array}$ \\
\hline $\begin{array}{l}\text { Cyclotella radiosa (Grun.) } \\
\text { Lemm. }\end{array}$ & $\begin{array}{l}\text { Appears in eutrophic conditions, was found in acidic or brackish water bodies, } \\
\text { neutral waters and very polluted lakes. }\end{array}$ \\
\hline Cymbella affinis Kutz. & $\begin{array}{c}\text { Indicator of very good ecological water quality, rarely found in calcerous, } \\
\text { oligotrophic lakes and rivers. }\end{array}$ \\
\hline
\end{tabular}




\begin{tabular}{|c|c|}
\hline Dominant species & Comment \\
\hline $\begin{array}{l}\text { Encyonopsis } \\
\text { microcephala (Grun.) } \\
\text { Krammer }\end{array}$ & $\begin{array}{l}\text { Can live in slightly acidic to alkaline conditions and low to medium electrolyte } \\
\text { conditions, if the habitat is oligosaprobic. Occurs in oligo- to slightly eutrophic } \\
\text { conditions. }\end{array}$ \\
\hline $\begin{array}{l}\text { Encyonema minutum } \\
\text { (Hilse) Mann }\end{array}$ & $\begin{array}{c}\text { Occurs in non-disturbed habitats (oligo- to mesotrophic conditions) with a medium } \\
\text { electrolyte content. }\end{array}$ \\
\hline $\begin{array}{l}\text { Cymbella tumida (Breb.) } \\
\text { Van Heurck }\end{array}$ & $\begin{array}{l}\text { Appears frequently but with small populations in meso- to eutrophic water bodies } \\
\text { with medium electrolyte content. }\end{array}$ \\
\hline Diatoma vulgaris Bory & $\begin{array}{l}\text { Appears in medium to high trophical conditions, also in lakes independent of } \\
\text { ecozone but more frequent in rivers. }\end{array}$ \\
\hline Eunotia praerupta Ehr. & $\begin{array}{c}\text { Mostly found in undisturbed, oligodistrophic habitats with low electrolyte content on } \\
\text { silicates. Sometimes appears numerously. }\end{array}$ \\
\hline $\begin{array}{l}\text { Fragilaria capucina } \\
\text { Desm. }\end{array}$ & $\begin{array}{l}\text { Most likely in oligotrophic to mesoeutrophic, slightly acidic to alkaline waterbodies } \\
\text { with low to medium electrolyte content but not very reliable. }\end{array}$ \\
\hline $\begin{array}{l}\text { Fragilaria crotonensis } \\
\quad \text { Kitton }\end{array}$ & $\begin{array}{l}\text { Mostly found in oligotrophic to slightly eutrophic, mostly slightly alkaline conditions } \\
\text { with medium electrolyte content. Decreases with increasing sewage input. }\end{array}$ \\
\hline $\begin{array}{l}\text { Staurosirella pinnata } \\
\text { (Ehr.) Williams \& Round }\end{array}$ & $\begin{array}{c}\text { Broad trophic tolerance, almost reaching over the whole spectrum. Negative reaction } \\
\text { to } \beta \text {-mesosaprobic conditions. Prefers non-acidic conditions. Also appears in } \\
\text { circumneutral conditions with low electrolyte content. }\end{array}$ \\
\hline $\begin{array}{l}\text { Gomphonema olivaceum } \\
\text { (Horn.) Breb. }\end{array}$ & $\begin{array}{l}\text { Mostly found in eutrophic, calcareous conditions with moderate electrolyte content. } \\
\text { Saprobic tolerance reaches from oligosaprobic to critical threshold between } \beta \text { and } \\
\qquad \alpha \text {-mesosaprobic conditions. }\end{array}$ \\
\hline $\begin{array}{l}\text { Gomphonema parvulum } \\
\text { (Kutz.) Kutz. }\end{array}$ & $\begin{array}{l}\text { Widely spread in oligotrophic habitats with low electrolyte contents. Mostly appears } \\
\text { in slightly acidic conditions in lakes. Appears together with acidophilic species. }\end{array}$ \\
\hline
\end{tabular}

Ecological characteristics of periphyton species which were present only at one or two research sites (based on [14-18])

\begin{tabular}{|c|c|}
\hline Ocasionally listed species & Occurrence and ecology \\
\hline Achnanthes exigua Grun. & $\begin{array}{c}\text { Lives in calcareous lakes, can survive with low light intensity, is able to live until } \\
\text { critical threshold ( } \beta-, \alpha \text {-mesosaprob). }\end{array}$ \\
\hline $\begin{array}{l}\text { Lemnicola hungarica } \\
\text { (Grun.) Round \& Basson }\end{array}$ & $\begin{array}{c}\text { Found in waters of higher trophic level and higher electrolyte contents. This } \\
\text { species is hypereutraphentic, alkaliphilous (mainly occurring at } \mathrm{pH}>7 \text { ) and has } \\
\text { low oxygen requirements. }\end{array}$ \\
\hline $\begin{array}{l}\text { Planothidium ellipticum } \\
\text { (Cleve) Edlund }\end{array}$ & $\begin{array}{l}\text { Ecological amplitude very broad, from neutral waters to alkaline, low electrolyte to } \\
\text {-rich, oligotrophic to polytrophic waters up to } \beta \text {-mesosaproben stress level. }\end{array}$ \\
\hline $\begin{array}{l}\text { Aulacoseira granulata } \\
\text { (Ehr.) Simon. }\end{array}$ & The classical indicator species of eutrophic water areas. \\
\hline $\begin{array}{l}\text { Caloneis silicula (Ehr.) } \\
\text { Cleve }\end{array}$ & Found in meso-eutraphentic waters with $\mathrm{pH}$ index above 7. \\
\hline $\begin{array}{l}\text { Encyonema cespitosum } \\
\text { Kutz. }\end{array}$ & $\begin{array}{l}\text { Found very often in eutrophic waters with moderate electrolyte content, but } \\
\text { tolerant of oligotrophic to eutrophic. }\end{array}$ \\
\hline $\begin{array}{l}\text { Encyonema elginense } \\
\text { (Krammer) Mann }\end{array}$ & $\begin{array}{l}\text { This freshwater species is widespread, which makes it a weak bioindicator of water } \\
\text { quality. It appears in low quantity of small specimens. }\end{array}$ \\
\hline $\begin{array}{c}\text { Eunotia intermedia }(\text { Krasske } \\
\text { ex Hustedt) Nörpel \& } \\
\text { Lange-Bertalot }\end{array}$ & $\begin{array}{l}\text { Autecology is the same as for majority of Eunotia species: occurrence in } \\
\text { minerotrophic, moderately acidic waters with low conductivity. }\end{array}$ \\
\hline $\begin{array}{l}\text { Eunotia bilunaris (Ehr.) } \\
\quad \text { Schaars. }\end{array}$ & $\begin{array}{l}\text { One of the most frequent and abundant Eunotia taxa living epiphytic in all regions } \\
\text { of Europe. Predominantly occurring in oligotrophic to dystrophic waters with low } \\
\text { electric conductance and low pH. However, resembling populations can also occur } \\
\text { in eutrophic ponds or lakes with moderately electrolyte content. }\end{array}$ \\
\hline
\end{tabular}


Benthic diatoms as valuable indicators of anthropogenic eutrophication in biomonitoring of ribbon lake 721

\begin{tabular}{|c|c|}
\hline Ocasionally listed species & Occurrence and ecology \\
\hline $\begin{array}{l}\text { Ulnaria capitata }(\text { Ehr.) } \\
\text { Compère }\end{array}$ & $\begin{array}{c}\text { This freshwater species is widespread, which makes it a weak bioindicator of water } \\
\text { quality. }\end{array}$ \\
\hline $\begin{array}{l}\text { Fragilaria } \\
\text { amphicephaloides } \\
\text { Lange-Bertalot }\end{array}$ & $\begin{array}{l}\text { This species is an indicator of excellent ecological quality. It has found in patchy } \\
\text { form in oligotrophic to mesotrophic lakes. It is rarely found in rivers. }\end{array}$ \\
\hline Fragilaria constricta Ehr. & $\begin{array}{l}\text { Stenotermic species of freshwaters, found often in the north parts of Europe. } \\
\text { Mainly occurring in oligotraphentic waters with pH index below } 7 .\end{array}$ \\
\hline $\begin{array}{l}\text { Fragilaria vaucheriae } \\
\text { (Kutz.) Petersen }\end{array}$ & $\begin{array}{l}\text { Still difficult to assess proliferation in the water types and ecological amplitude due } \\
\text { to difficult identification. }\end{array}$ \\
\hline $\begin{array}{l}\text { Gomphonema angustatum } \\
\text { (Kutz.) Rabenhorst }\end{array}$ & $\begin{array}{l}\text { Secured findings as to the identity, in circumneutral waters, such as ponds or lakes } \\
\text { with dead plant materials. }\end{array}$ \\
\hline $\begin{array}{l}\text { Gomphonema angustum } \\
\text { Agardh }\end{array}$ & $\begin{array}{l}\text { Typical taxon of very hard waters, there almost always individual-rich. Where such } \\
\text { habitats are absent, G. angustum is substituted with other, perhaps similar } \\
\text { Gomphonema species. }\end{array}$ \\
\hline Gomphonema augur Ehr. & $\begin{array}{l}\text { Distribution and ecology difficult to characterize. The most abundant in alkaline, } \\
\text { nutrient-rich lakes and larger rivers, saprobie-tolerant up to the critical load factor } \\
\qquad(\beta-, \alpha \text {-mesosaprob). }\end{array}$ \\
\hline $\begin{array}{l}\text { Mastogloia smithii Thwaites } \\
\text { ex Smith }\end{array}$ & $\begin{array}{l}\text { On the European Baltic coasts widespread brackish water kind, not seldom, } \\
\text { however, also in lakes of the interior with middle and higher electrolyte content. }\end{array}$ \\
\hline $\begin{array}{c}\text { Mayamaea agrestis } \\
\text { (Hustedt) Lange-Bertalot }\end{array}$ & This freshwater species is found in hypertrophic waters. \\
\hline Navicula dicephala Ehr. & The most abundant in eutrophic waters with moderate electrolyte content. \\
\hline $\begin{array}{l}\text { Placoneis gastrum (Ehr.) } \\
\text { Meresch. }\end{array}$ & $\begin{array}{c}\text { Found in eutraphentic waters, mostly lakes. Has high oxygen requirements (above } \\
75 \% \text { ). Mainly occurring at } \mathrm{pH}>7 \text {. The habitats are meso- to eutroph and } \\
\text { oligosaprob. }\end{array}$ \\
\hline $\begin{array}{l}\text { Pinnularia gibba (Ehr.) } \\
\text { Muller }\end{array}$ & $\begin{array}{l}\text { Hypereutraphentic, cosmopolitan, in places abundant in waters with low to average } \\
\text { electrolyte content, especially in spring-like locations. }\end{array}$ \\
\hline $\begin{array}{l}\text { Rhoicosphaenia abbreviata } \\
\text { (Ag.) Lange-Bertalot }\end{array}$ & $\begin{array}{c}\text { Cosmopolitan species inhabiting freshwaters with moderately high electrolyte } \\
\text { content and brackish-waters. }\end{array}$ \\
\hline $\begin{array}{l}\text { Surirella minuta (Breb.) } \\
\text { Kutz. }\end{array}$ & $\begin{array}{l}\text { Because of preferences toward waters with smaller electrolytic conductivity, this } \\
\text { species is use as an indicator of low thropic state-mesotrophy, weak eutrophy. }\end{array}$ \\
\hline
\end{tabular}

\section{Discussion}

Periphyton is an ecological group of organisms attached to abiotic structures submerged in water such as stones, and biotic structures like macrophytes. Phytoperiphyton is not only the group of organisms responsible for primary production in water but also the group which is a sensitive indicator for changes in parameters of water quality [3, 13]. Periphyton is constantly attached to its substrate, thus the structure of periphytic community reacts to both physical and chemical changes in water quality. Periphytic communities, mainly diatoms, are very good biological indicators for various reasons: naturally big group of species, fast reaction time to changes in habitat conditions, well documented tolerance spectra, and sensitivity of individual species to changes in water quality.

In order to evaluate the ecological state of Lake Durowskie, taxonomical structure and quantity of individual diatom species were studied. Results were, then, compared to referral conditions set for lakes. In the research conducted periphytic diatoms were collected along the shoreline of Lake Durowskie. Studies concerning its taxonomical structure and abundance of periphytic communities from 12 sites are shown jointly. Periphyton analyses 
demonstrated the presence of 104 diatom taxa which can prove useful in determining the ecological state of water in various places along the shoreline of the lake (Table 3).

Studies conducted in years 2010-2018 showed that the process of changes in the lake ecosystem, which had started in 2009 with the beginning of restoration measures, is visible in both physicochemical parameters and biological ones. Transparency of water, which was noted at ca. $1 \mathrm{~m}$ in 2009 increased to 2,5 $\mathrm{m}$ in 2018. Concentrations of ammonium nitrogen, nitrates and dissolved phosphates were also noted at lower levels, which proves the increasing role of nitrification connected with good oxygen conditions in water $[4,6,19]$. Dissolved oxygen refers to the level of free, non-compound oxygen present in water or other liquids. It is an important parameter in assessing water quality because of its influence on the organisms living within a water body. A too high or too low dissolved oxygen level might harm aquatic life and affect water quality. The reason for higher dissolved oxygen levels recorded at the beaches and at the outflow could be due to the constant mixing of the water by kayaks, speed boats, and other water vehicles as well as swimming in these areas. Higher transparency of water also caused better development of submerged plants in the lake's littoral zone. Improvement of water quality in littoral zone may be noted due to occurrence of red algae species Hildenbrandia rivularis (Liebm.) Agardh [20], which indicates good oxygenated water. Positive changes in phytobenthos composition can be seen in the higher ratio of Pantocsekiella ocellata (Pant.) Kiss \& Acs, which is an indicator species for less fertile waters with low amounts of organic matter and high level of oxygenation [4, 13]. Obtained results also suggest the type of land used in the direct catchment area may significantly impact the diversity of species. The northern part of the lake, whose direct catchment area has a high ratio of forests, was characterized by better quality of water comparing to the southern part located in the urban area, which was reflected in the structure of the phytobenthic community and the values of the Diatom Index.

In 2010, the Diatom Index indicated that the northern part of the lake is characterized by poor quality of water $(0.28-0.29$; poor state), while the water quality of the rest of the lake was characterized to be moderate $(0.36-0.54)$. Moreover, for three of the sites in the southern part of the lake, the values noted for the DI $(0.51 ; 0.52 ; 0.54)$ were very close to the threshold between a moderate and a good state of water.

In the following year, the Diatom Index showed decreasing tendency in all of the analyzed sites. Both at the northern and southern sides of Lake Durowskie the quality of water was indicated to be poor (Table 2), with the exception of two sites in which said quality was indicated to be moderate. Observed changes meant that the water state was decreased in 2011. Only two of the eight sites stayed at similar water state to previous year. One of the reasons for these changes can be the increase in the water level caused by intense rainfall together with higher inflow from the direct catchment area mainly in the form of surface runoff but also with inflow from Struga Golaniecka river. Moreover, turbulence of water in the littoral zone caused by wind can also increase the amount of nutrients available for phytobenthos. The improvement of aerobic conditions in the lake can be seen in the greater share of species with preference to highly oxygenated water in periphytic communities.

Since 2012 indicators of very good and good oxygenation of water has been noted in the lake. Those species, such as Achnanthidium minutissimum (Kutz.) Czarnecki, show increase in the total number of cells. Indicators of bad or poor water quality stayed at the same numerical level as observed previously or noted a slight decrease. In the case of 
Gomphonema parvulum (Kutz.) Kutz., which indicates poor quality of water, it was found abundant only at the southern sites of the lake (e.g. site 4) with only a few individuals noted at rest of the sites. The reason for its high abundance at site 4 may be due to the use of this part of the shoreline as wild beach at which people allow their dogs to get in the water, thus increasing the turbulence of water at this site. Fine seston suspension and pollution brought from the shore drop to the bottom, and then undergo decomposition by reducers, with the use of oxygen, in the surface layer of bottom sediments. The Diatom Index values increased from poor state to moderate at two sites located in the northern part of Lake Durowskie (Table 2).

These results point to a significantly lower inflow of pollutants than in previous years, as well as to a gradual improvement of the ecological state of this part of the lake. In the central and southern parts of the lake the Diatom Index stayed at poor level or balanced between poor and moderate ecological states. However, even though there was no meaningful increase in the DI value, a drastic decrease was also not observed. The increase in abundance od periphytic species adapted to very good oxygen conditions additionally emphasized success of restoration measures carried out since 2008.

At some of the sites (e.g. 6, 7,9) the ecological state of water has been increasing since 2010-2011. At the same time, at the other sites the ecological state of water has been visibly decreasing. It is particularly well visible for site 3 where the ecological state has been decreasing yearly and reached the poor state in 2014, which was the lowest value since the beginning of monitoring 5 years prior. Analyses of the Diatom Index values showed clear diversification between the northern and southern parts of Lake Durowskie. Mean values for sites located in the northern (sites 4, 5, 6, 7, 8) and southern parts (sites 1,2,3) showed clearly that ecological state is better in the northern part (Table 2). The biggest biodiversity of a phytobenthic community was noted in the middle of the lake's length, near the Aerator 2. This observation suggests that phytobenthic community structure changed rapidly in the part of the lake which is characterized by low human impact. From these results we may conclude with high probability that the worse ecological state of the southern part of the lake is connected with higher anthropogenic pressure. In the southern part the decrease in biodiversity occurs as an effect of recreational activities as well as the erosion of the shoreline and the direct catchment area, and fishing together with using ground bait and motorboats. Lower values of the Diatom Index in the southern part of the lake noted since 2014 may serve as confirmation of such interactions, especially for the south-western part of the lake. In the northern part, even with the influence of river Struga Golaniecka, the DI values point to a moderate ecological state, in opposition to the southern part where the Diatom Index indicates a poor water state. Due to the northern part's direct catchment area being composed mainly by forests, in the northern part of the lake submerged macrophytes are less exposed to damage which results in higher absorption of nutrients by aquatic vegetation. Occurrence of stonewort Chara tomentosa L. in the southern part of the lake also confirms a better ecological state of the lake's water in the north [4].

Diatom Index calculated for the years 2016-2018 suggests that anthropogenic pressure plays a key role in shaping of diatom communities. The lowest values of DI were noted on the sites with highest anthropogenic pressure (sites 1 and 2). During the last years of investigations (2017-2018) the Trophic Diatom Index (TDI) showed more or less the same conditions as in previous years, showing poor to moderate state of water. The remarkable drop of TDI at Site 1 (Beach 1 ; 2017) has shown possible negative effects from anthropogenic activities and input causing the upwelling of water and mobilization of 
nutrients from the bottom of the lake. The south-eastern part of the lake, where a public beach is located, is the most densely populated area for recreation and according to the TDI showed in 2017, this area remains in a bad/poor state of water. However, some of the parts of the lake begin to improve, implying the restoration has had positive effect. Moreover, at site 4, where the area has recorded an increase in Hildenbrandtia rivularis (Liebm.) Agardh (red algae) population, the TDI also shows the greatest improvement. At site 1 in 2018 there was an increase in quality (Table 2) and this could be attributed to the fact that two new macrophyte species were found around the site this year. Both Chara contraria Braun ex (Kutz.) and Nitellopsidetum obtuse Allen help the water quality so there is a correlation between these species being found and the increase in water quality at site 1 [21]. According to the index, the average composition of species is similar. Compared to last year's results, the number of oxygenated groups increased. It means the state of littoral water has improved. It occurred with almost the same number between the northern and southern parts of the lake (Fig. 6). For the trophic preference, the eutrophic species still dominate at every site (Fig. 7), and compared to last year's results, the number of those species increased in several areas. However, from the result of $\mathrm{pH}$ preference, the alkaliphilous species are dominant in all parts of the lake. The comparison of results from the northern and southern parts of the lake shows that the conditions are very similar, as shown on Figure 8. Results imply that the water from both the north and the south tends to be well oxygenated, alkali and eutrophic.

\section{Conclusions}

The eutrophication of water of Lake Durowskie is probably due to pollution transfer from other nearby regions through inflow. Erosion and runoff along the bank can also deteriorate its condition. The highest anthropogenic pressure occurs in the southern part of the lake which is situated in Wagrowiec. On this side of the lake the littoral zone is very narrow and the shoreline is spotted with fishing platforms, piers for boats and also a beach, all of which pose high recreational value for the people of Wagrowiec as well as for tourists. Field observation by counting the number of fishing stands and swimming places around the lake may point to other drivers of pollution. With almost 103 fishing sites, and 14 swimming sites, the amount of left-over fish bait and the surface of open soil should be considered.

Results obtained from particular indicators do not always show the same tendency for changes in the environment, which is caused mainly by the fact that the ecosystem is a very complex structure and biotic and abiotic factors inside the trophic net influence various groups of organisms [22, 23]. Nevertheless, obtained results show in almost full picture changes in the environment which could not be evaluated on the basis of random physicochemical or hydrobiological measurements. Different results from the evaluation of different parameters of the ecosystem occur because of different sensitivity and reaction time to environmental changes for given ecological groups of organisms. Ecological changes can be most quickly observed in the quality of water and phytoplankton communities, then, in microbenthos and, then, in macrophytes and macrozoobenthos [1].

\section{Acknowledgements}

The authors thank all participants of the Summer Schools for their contribution to the study of changes in water quality and composition of organisms in Lake Durowskie in the 
Benthic diatoms as valuable indicators of anthropogenic eutrophication in biomonitoring of ribbon lake 725

years of restoration. Each year the research was funded by the Mayor of Wagrowiec, for which the authors are grateful to him.

\section{References}

[1] Directive 2000/60/EC. Establishing a framework for community action in the field of water policy. European Commission PE-Cons 3639/1/100 Rev 1. Luxembourg: Commission to the European Communities; 2000. https://eur-lex.europa.eu/legal-content/EN/ALL/?uri=CELEX:32000L0060.

[2] Schaumburg J, Schranz C, Stelzer D, Hofmann G. Action Instructions for the Ecological Evaluation of Lakes for Implementation of the EU Water Framework Directive: Macrophytes and Phytobenthos. Bavarian Environment $\quad 2007 . \quad$ http://www.daba.lv/grozs/HidroBiologjijas/Kalme/ LVAF_projekta_\%20materiali/Ekol_klasifik_dok/GE_biolog_el/instruction_protocol_lakes.pdf.

[3] Szczepocka E, Żelazna-Wieczorek J. Diatom biomonitoring - scientific foundations, commonly discussed issues and frequently made errors. Oceanological Hydrobiol Stud. 2018;47(3):313-25. DOI: 10.1515/ohs-2018-0030

[4] Gołdyn R, Messyasz B, Domek P, Windhorst W, Hugenschmidt C, Nicoara M, et al. The response of Lake Durowskie ecosystem to restoration measures. Carpathian J Earth Environ Sci. 2013;8(3):43-8. Available from: https://www.researchgate.net/publication/258206386_The_response_of_Lake_Durowskie_ecosystem_ to_restoration_measures.

[5] DeNicola DM, Kelly M. Role of periphyton in ecological assessment of lakes. Freshwater Sci. 2014;33:619-38. DOI: 10.1086/676117.

[6] Kowalczewska-Madura K, Dondajewska R, Gołdyn R, Kozak A, Messyasz B. Internal phosphorus loading from the bottom sediments of a dimictic lake during its sustainable restoration. Water Air Soil Pollut. 2018;229(8):280. DOI: 10.1007/s11270-018-3937-4.

[7] Battarbee RW, Kneen MJ. The use of electronically counted microspheres in absolute diatom analysis. Limnology Oceanography. 1982;27:184-8. DOI: 10.4319/lo.1982.27.1.0184.

[8] Lange-Bertalot H. 85 New Taxa and Much More than 100 Taxonomic Clarifications Supplementary to Süsswasserflora von Mitteleuropa. Berlin, Stuttgart: VEB Gustav Fischer, Verlag; Bibl Diatom, 1993;2(1-4):p. 760. ISBN: 3443570186.

[9] Lange-Bertalot H. Navicula sensu stricto, 10 genera separated from Navicula sensu lato, Frustulia. In: Lange-Bertalot H, editor: Diatoms of Europe. Diatoms of the European inland waters and comparable habitats. ARG, Gantner Verlag KG; 2001;2:p.526. ISBN: 9783904144780.

[10] Lange-Bertalot H, Metzeltin D, editors. Oligotrophie - Indikatoren. Iconographica Diatomologica. Annotated Diatom Micrographs. Königstein: Koeltz Scientific Books; 1996. ISBN: 9783874293860.

[11] Lange-Bertalot H, Hofmann G, Werum M, Cantonati M. Freshwater Benthic Diatoms of Central Europe: Over 800 Common Species Used in Ecological Assessment. Schmitten-Oberreifenberg: Koeltz Botanical Books; 2017; p.942. ISBN: 9783946583066.

[12] Guiry MD, Guiry GM. AlgaeBase. World - Wide Electronic Publication. Galway: National University of Ireland; 2018. http://www.algaebase.org.

[13] Van Dam H, Martens A, Sinkeldam J. A coded checklist and ecological indicator values of freshwater diatoms from the Netherlands. Netherlands J Aquatic Ecol. 1994;28(1):117-33. DOI: 10.1007/BF02334251.

[14] Krammer K, Lange-Bertalot H. Bacillariophyceae. 1. Naviculaceae. In: Ettl H, Gerloff J, Heynig H, Mollenhauer D, editors. Süsswasserflora von Mitteleuropa. Stuttgart, New York: VEB Gustav Fischer, Verlag; 1986;2(1):p. 876. ISBN: 9783827426154.

[15] Krammer K, Lange-Bertalot H. Bacillariophyceae. 3. Centrales. In: Ettl H, Gerloff J, Heynig H, Mollenhauer D, editors. Süsswasserflora von Mitteleuropa. Stuttgart, New York: VEB Gustav Fischer, Verlag; 1991;2(3):p. 576. ISBN: 3827419875.

[16] Krammer K, Lange-Bertalot H. Bacillariophyceae. 4. Achnanthaceae, Kritishe Erganzungen zu Navicula (Lineolatae), Gomphonema Gesamtliteraturverzeichnis Teil 1-4 [second revised edition]. In: Ettl H, Gerloff J, Heynig H, Mollenhauer D, editors. Süsswasserflora von Mitteleuropa. Stuttgart, New York: VEB Gustav Fischer, Verlag; 2004; 2(4):p.468. ISBN: 3437306642.

[17] Krammer K. Diatoms of Europe. Diatoms of European Waters and Comparable Habitats. Cymbella. A.R.G. Gantner Verlag. Kommanditgesellschaft, Ruggel, 2002; p. 584. ISBN: 9783904144247.

[18] Lange-Bertalot H, Wojtal AZ. Diversity in species complexes of Placoneis clementis (Grunow) Cox and Paraplaconeis placentula (Ehr.) Kulikovskiy. Lange-Bertalot Metzeltin, Nova Hedwigia. 2014;143:403-20. DOI: $10.1127 / 1438-9134 / 2014 / 021$. 
[19] Dondajewska R, Kowalczewska-Madura K, Gołdyn R, Kozak A, Messyasz B, Cerbin S. Long-term water quality changes as a result of a sustainable restoration - a case study of Dimictic Lake Durowskie. Water. 2019;11(3):616. DOI: 10.3390/w11030616.

[20] Eloranta P, Kwandrans J. Freshwater Red Algae. Rhodophyta. Identification Guide to European Taxa. Saarijärven Offset Oy. Norlinia, 2007;15:3-103. ISBN: 9789521039461.

[21] Van der Valk, The Biology of Freshwater Wetlands. Oxford and New York: Oxford University Press; 2012. ISBN 9780199608959.

[22] Blanco S, Cejudo-Figueiras C, Tudesque L, Bécares E, Hoffmann L. Ector L. Are diatom diversity indices reliable monitoring metries? Hydrobiologia. 2012;695:199-206. DOI: 10.1007/s10750-012-1113-1.

[23] Vilmi A, Karjalainen SM, Landeiro VL, Heino J. Freshwater diatoms as environmental indicators: evaluating the effects of eutrophication using species morphology and biological indices. Environ Monit Assess. 2015;187:243. DOI: 10.1007/s10661-015-4485-7. 